\title{
Study of the fatigue life and weight optimization of an automobile aluminium alloy part under random road excitation
}

\author{
A. Saoudi, M. Bouazara* and D. Marceau \\ Department of Applied Sciences, University of Quebec at Chicoutimi, Saguenay, (Qc), Canada G7H 2BI
}

Received 3 April 2008

Revised 1 March 2009

\begin{abstract}
Weight optimization of aluminium alloy automobile parts reduces their weight while maintaining their natural frequency away from the frequency range of the power spectral density (PSD) that describes the roadway profile. We present our algorithm developed to optimize the weight of an aluminium alloy sample relative to its fatigue life. This new method reduces calculation time; It takes into account the multipoint excitation signal shifted in time, giving a tangle of the constraint signals of the material mesh elements; It also reduces programming costs. We model an aluminium alloy lower vehicle suspension arm under real conditions. The natural frequencies of the part are inversely proportional to the mass and proportional to flexural stiffness, and assumed to be invariable during the process of optimization. The objective function developed in this study is linked directly to the notion of fatigue. The method identifies elements that have less than $10 \%$ of the fatigue life of the part's critical element. We achieved a weight loss of 5 to $11 \%$ by removing the identified elements following the first iteration.
\end{abstract}

Keywords: Automobile, fatigue, vibration, aluminium, random road, optimization

\section{Introduction}

In this study, we examine the fatigue life and weight optimization of a real, complex aluminium alloy part: the lower suspension arm of a vehicle, an essential component of the suspension system. Haiba et al. [1] have developed an objective function to optimize the weight of a mechanical component without affecting natural frequencies and fatigue life distribution. They constructed a rejection ratio from the fatigue life of all elements and the critical element one of the mesh. They calculated the fatigue life of each mesh element from the experimental W öhler curve extrapolation. Wöhler curve extrapolation takes into account a single parameter, in this case the stress variation. Elmarakbi et al. [2] used an energy model taking into consideration two parameters: stress and strain. They studied the validity of the multiaxial fatigue criterion based on strain energy density, but did not propose a rejection ratio for weight optimization. Instead, they provided a model to assess the predictive capabilities of several theories of multiaxial fatigue, such as bending, twisting or testing combinations of flexion-torsion made by SAE (Society of Automotive Engineers). They compared the strain energy density of the multiaxial to the uniaxial case, as the two tensors having the same order, zero. March [3] based his work on the same criterion as Elmarakbi et al. [2], the energy density of cracking. He compared results of fatigue tension-torsion tests with the strain energy density criterion. He also proposed a model for predicting the fatigue life of rubber particles using two approaches. The first focused on the duration of crack initiation giving parameters such as stress status and strain that define a material point. The other approach focused on crack propagation using the Griffith criterion.

\footnotetext{
${ }^{*}$ Corresponding author. E-mail: mbouazar@uqac.ca.
} 
In addition to Elmarakbi et al.'s [2] deterministic model used in the present study, there are other models in the literature that cover stochastic aspects of the fatigue phenomenon. For example, Asok et al. [4] proposed a stochastic model to study fatigue crack propagation in metallic materials, a relatively recent research area. Asok and Ravindra [5] used a stochastic model based on experimental observations and a deterministic structure model of state space with two state variables, crack length and stress opening. They applied an equation governing fatigue crack propagation in a random aspect similar to what was originally developed by Paris and Erdogan in 1963. In contrast, de-Andrées et al. [6], used a cohesive element law for predicting part endurance where a gradual loss of elasticity creates new areas as separation increases.

The basic model of an oscillating system is used to study the natural frequency changes and to identify multiple cracks in a mechanical system. The oscillating system is composed of mass $m$ and spring $k$. Newton's second law allows us to set the natural frequency as:

$$
\omega_{n}^{2}=\frac{k}{m}
$$

Next, the transverse vibration at the end of a bending beam is included. This type of beam behaviour is similar to an automobile rear suspension and to the wings of some aircraft. The mass $m$ at the tip of the beam will oscillate with natural frequency given in Eq. (2).

$$
\omega_{n}=\sqrt{\frac{k}{m}}=\sqrt{\frac{3 E I}{m l^{3}}}
$$

where $l$ is the length of the beam, $E$ is the elastic (Young's) modulus of the beam, and $I$ is the (area) moment of inertia of the cross-sectional area [7].

Athanasios et al. [8], have used changes in natural frequency to identify the position and depth of multiple cracks in a bending beam. When cracks are present in a structure, such as a beam, the natural frequency of vibrations decreases [8]. Stephen and Zhang [9] studied a repetitive beam structure under loading and vibration coupling torsion-tension. They used eigenvalue analysis of a single cell in a repetitive structure of planar beams in dynamic theories for flexural vibrations to predict natural frequencies of vibration. Coupling torsion-flexion was studied on a Timoshenko beam charged with a rigid mass at one end [10]. The Timoshenko beam model is more precise than the Euler-Bernoulli beam model. It can be applied to thin and thick beams in both high and low frequency vibration fields, thus the results obtained are more significant. Hamed and Frostig [11] proposed using a natural frequency formula for an axially compressed and suspended beam:

$$
\omega_{n}=\frac{n \pi}{L_{\text {beam }}} \sqrt{\frac{1}{m_{\text {beam }}}\left[E I\left(\frac{n \pi}{L_{\text {beam }}}\right)^{2}-N\right]}
$$

Where $E I$ is the beam flexural stiffness, $L_{\text {beam }}$ is the beam length, $m_{\text {beam }}$ is the beam mass per unit length, $n$ is the mode number and $N$ is the axial compressive strain.

The natural frequency of the cable under tension is:

$$
\omega_{n}=\frac{n \pi}{L_{\text {cable }}} \sqrt{\frac{T}{m_{\text {cable }}}}
$$

Where $L_{\text {cable }}$ is the cable length, $m_{\text {cable }}$ is the mass per unit length and $T$ is the tensile strain intensity. Kyprianou et al. [12] have solved the problem of reverse modified structures to determine beam cross-section dimensions, especially when it is added to the original structure, controlling natural frequencies or resonance.

In the present paper, we predict a part's fatigue life by developing a multiaxial elasto-plastic numerical model. Using the aluminium alloy 7075-T6 of known mechanical properties, we simulate the fatigue rupture prediction with the commercial finite element software Abaqus. The number of cycles to failure is calculated using the uniaxial equivalent of the multiaxial model. Also, the natural frequencies of the part are calculated. The lower vehicle suspension arm of a quarter vehicle suspension system is studied in a critical case, where the part is embedded in the suspension joint. The stress state underestimates true fatigue life and hence it gives shorter and safer values. The lower vehicle suspension arm weight is optimised by an objective function, which makes it light and safe, and its natural frequencies far from the frequency range caused by road irregularity. 


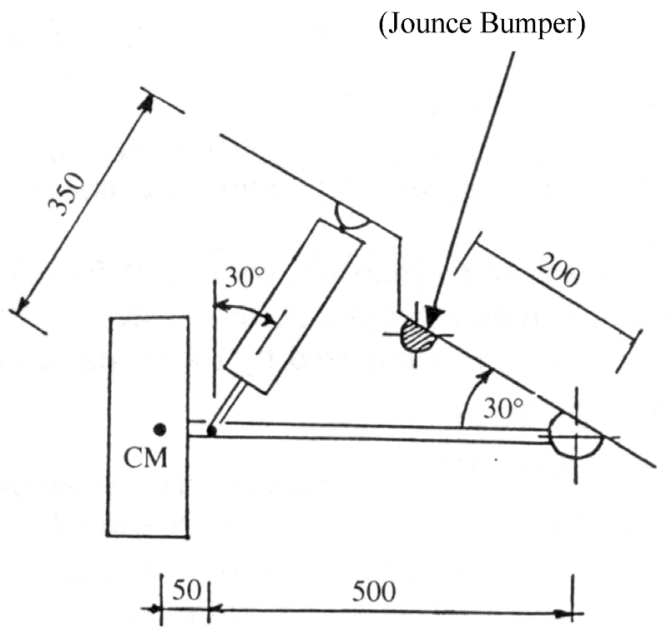

(a)

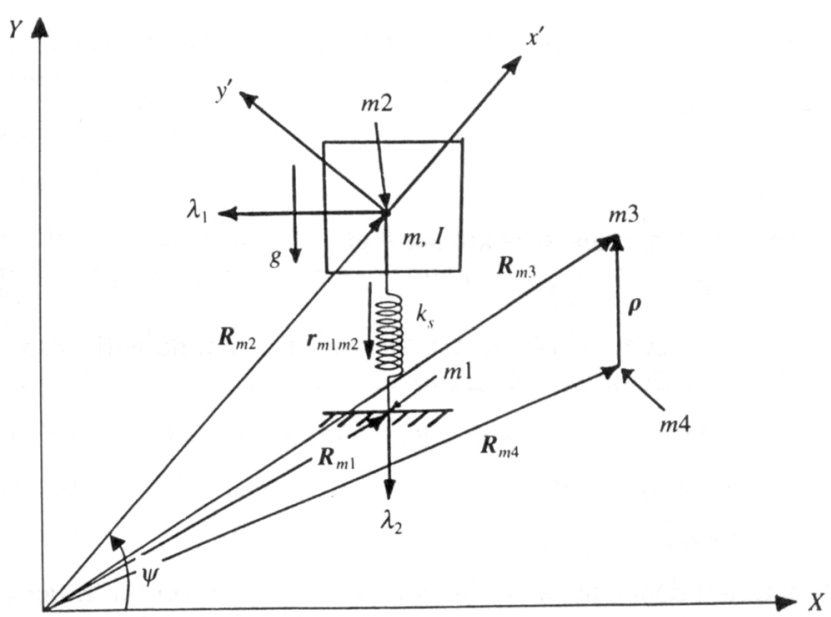

(b)

Fig. 1. Quarter vehicle McPherson suspension system.

In this study, a dynamic vehicle suspension system model and a numerical model using the commercial finite element are developed. The results are filtered to extract the critical elements in the elasto-plastic case. The partial and total fatigue life of the part in multiaxial model is calculated from the uniaxial criterion equivalent case. The natural frequency of the embedded piece is also calculated. We end by developing an objective function to optimize the weight of the lower suspension arm.

The excitation is analytically calculated from the power spectral density (PSD) acting on the lower suspension arm of a vehicle through a tire of stiffness constant $K_{p}$, where the return chain is made up of a linear shock absorber and a linear spring. To evaluate the dynamic behaviour of the suspension system and the roadway profile model, Rahnejat [13] studied the dynamics of the Macpherson suspension system in a quarter vehicle. The spring and the shock absorber are represented as a single element with stiffness constant $k_{s}$ (Fig. 1). In fact, Rahnejat proposes a simple model where the mass and inertia are represented by parameters $m$ and $I$ respectively. The model developed in this study is based on vertical motion and road excitation.

\section{Road profile model development}

In the model shown in Fig. 2, the partial stability of the vehicle is ensured by a suspension control system. The action chain of the system has a tire of stiffness constant $k_{p}$. The return chain in negative feedback has a spring of stiffness $k_{s}$ assembled with a shock absorber $C_{s}$. The excitation force $F_{2}$ of the road irregularity is balanced through the tire by the $F_{1}$ negative feedback. The $F_{1}$ negative feedback brings back the suspension arm to its place of equilibrium linearly. For cases of small disturbances where the elastic behaviour of the material dominates, it produces a state of the low stress. So it's possible to write:

$$
\frac{x_{1}}{L_{1}}=\frac{x_{2}}{L_{2}} \Rightarrow x_{1}=\frac{L_{1}}{L_{2}} x_{2}
$$

$\mathrm{x}_{2}$ is the deflection from the equilibrium position caused by the road profile, allows the calculation of displacement $\mathrm{x}_{1}$ by using Eq. (5). Unsprung mass, such as the mass of the spring and the shock absorber is negligible compared to the dynamic stress brought into play. The suspension parameters are given in Table 1.

The forces applied to the lower suspension arm by a quarter vehicle are: $F_{2}=K_{p} x_{2}$ exerted on the tire by $x_{2}$ and $F_{1}=K_{s} x_{1}+C_{s} x_{1}$ negative feedback force exerted by the spring and the shock absorber.

Knowing the PSD experimental values, $x_{2}$ and consequently $F_{2}$ can be calculated. Therefore, Eq. (5) enables us to obtain the $x_{1}$ spectrum. Once $x_{1}$ is known, the complete determination of the $F_{1}$ values is done. The road 
Table 1

Suspension parameters

\begin{tabular}{ccccc}
\hline Sprung mass $m_{s}$ & Unsprung mass $m_{1}$ & Spring $K_{s}$ & Shock absorber $C_{s}$ & Tire $K_{p}$ \\
\hline $240 \mathrm{~kg}$ & $36 \mathrm{~kg}$ & $16000 \mathrm{~N} / \mathrm{m}$ & $1000 \mathrm{~N} . \mathrm{s} / \mathrm{m}$ & $160000 \mathrm{~N} / \mathrm{m}$ \\
\hline
\end{tabular}

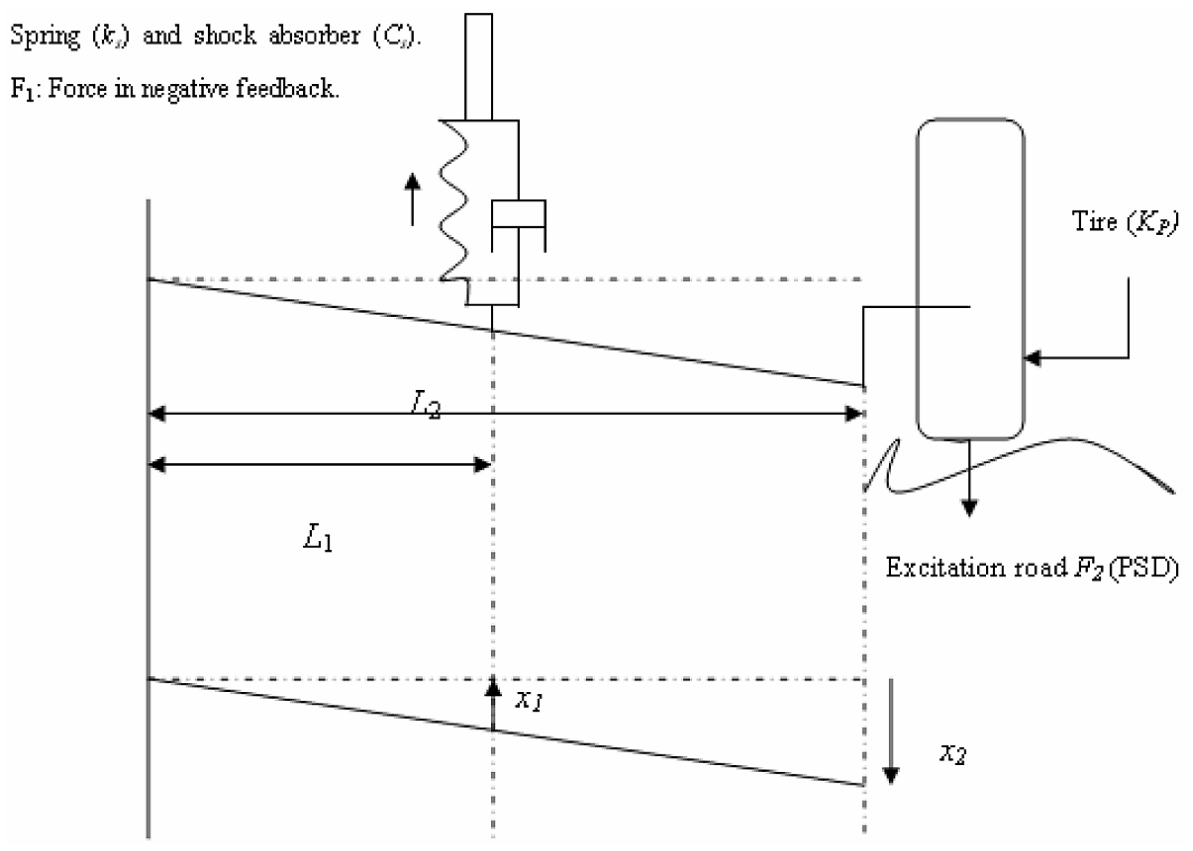

Fig. 2. Control of the lower suspension arm of a vehicle.

profile spectrum is a function of the vehicle speed, which is obtained from the PSD. Normalisation of the road profile spectrum is necessary to keep the same frequency band and to be able to transform it into force excitation. The factor, which must be multiplied by the values of the PSD, depends on the numerical values of the suspended mass, the tire stiffness constant, the shock absorber coefficient, and the damping ratios of the shock absorber and the unsprung mass. In this work, the numerical value of this factor is estimated to be $0.025 . x_{1}$ is given numerically by:

$$
x_{1}=\frac{x_{1}(t+\Delta t)-x_{1}(t)}{\Delta t}
$$

The excitation caused by the road profile has a random nature. In this work the road profile is estimated from the PSD. The road profile is defined by function $X(t)$. Assuming the road surface is a random, stationary, Gaussian and centered process i.e. all its statistical properties are invariable for any change of the argument $t$, that the law of distribution of variable $X(t)$ is a Gaussian law, and the average of $X(t)$ for any $t$ pertaining to [0, T] is null. The stationary random process and the Gaussian law $X(t)$ can be regarded as a periodic function in time $t$, of amplitude $\alpha$, circular frequency $\omega$ and phase $\phi$ [14]. The PSD calculation was performed by M. Bouazara. The function $X(t)$ is calculated using the PSD (Fig. $3 \mathrm{a}$ and $3 \mathrm{~b}$ ).

The road spectrum thus obtained corresponds to a vertical random displacement. However, this study takes into account the direct excitation by a random dynamic force. Thus it is necessary to transform the random displacement into a random force while keeping the same pulsation or band frequency. Therefore the minor road spectrum is normalised. The values of this spectrum are multiplied by a common factor estimated from the statistical data. For a minor road, the speed of the vehicle is lower than $75 \mathrm{~km} / \mathrm{h}(21 \mathrm{~m} / \mathrm{s})$. At this speed, a $960 \mathrm{~g}$ vehicle has a momentum equal to $20160 \mathrm{~kg} . \mathrm{m} / \mathrm{s}$. Thus in 0.5 second, the maximum force excitation is estimated at $40320 \mathrm{~N}$. This force is distributed equally on the four quarters of the vehicle's suspension system. The vehicle weight opposes around $3750 \mathrm{~N}$ in the return chain to the maximum $4000 \mathrm{~N}$ random excitation value. The maximum value of the force of excitation through a tire of stiffness $160000 \mathrm{~N} / \mathrm{m}$ is about $4000 \mathrm{~N}$. Figure $4 \mathrm{a}$ and $4 \mathrm{~b}$ shows the comparison between 
Table 2

Elasto-plastic behaviour of some aluminium alloys

\begin{tabular}{lcccc}
\hline Alloy & Code & Ultimate limit $\sigma_{u}(\mathrm{MPa})$ & Yield stress $\sigma_{e}(\mathrm{MPa})$ & Ratio: $\frac{\sigma_{u}-\sigma_{e}}{\sigma_{u}}$ en $\%$ \\
\hline Aluminium alloy & 2024-T351 & 455 & 379 & 16.70 \\
& 2024-T4 Al & 476 & 303 & 36.34 \\
& 7075-T6 Al & 578 & 469 & 18.85 \\
\hline
\end{tabular}

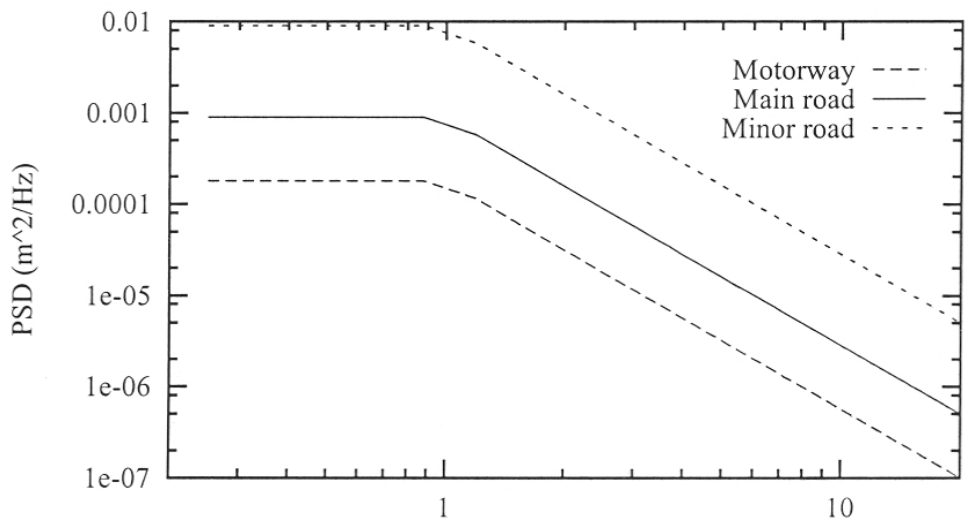

(a) Power spectral density (PSD) as a function of frequency $(\mathrm{Hz})$.

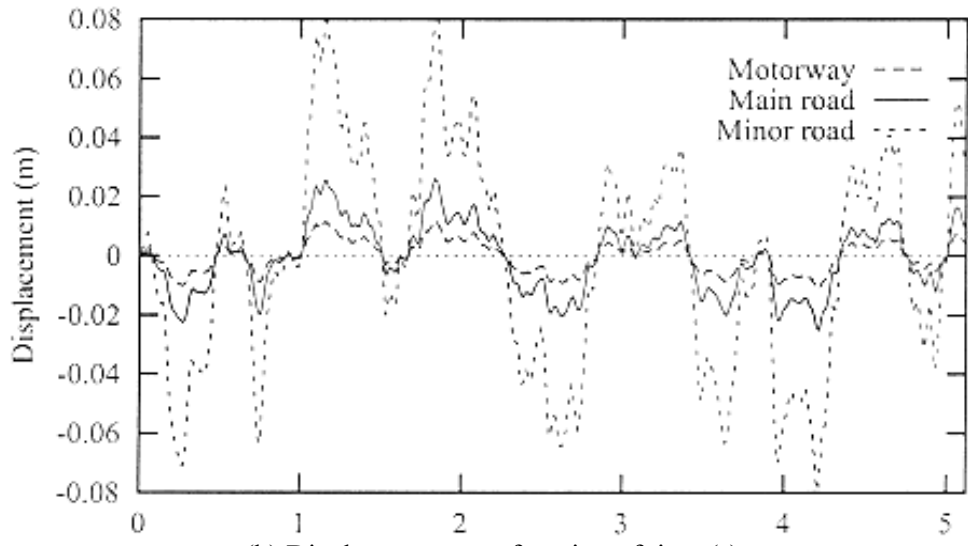

(b) Displacement as a function of time (s).

Fig. 3. Road profile.

the damped and undamped spectrums. It is also an illustration of the opposition of phases between the $F_{2}$ excitation and the $F_{1}$ force of the return chain in the vehicle suspension control system.

\section{Elasto-plastic numerical model development}

Aluminium alloys have an elasto-plastic behaviour with up to $36 \%$ plasticity (Table 2). Gbadebo $[15,16]$ proposed an analytical solution for the elasto-plastic case where the matrix of the total tensor deflection increments is the sum of the elastic and plastic contributions. They extrapolated the nonlinear uniaxial behaviour of the material in the multiaxial case to calculate the strain energy density. This approach is the same as that used in the commercial finite element software Abaqus.

The model takes into account the two cases of nonlinearity: non-geometric linearity (variation of the geometric configuration of the system in time) and material nonlinearity which expresses the elasto-plastic coupling. The 


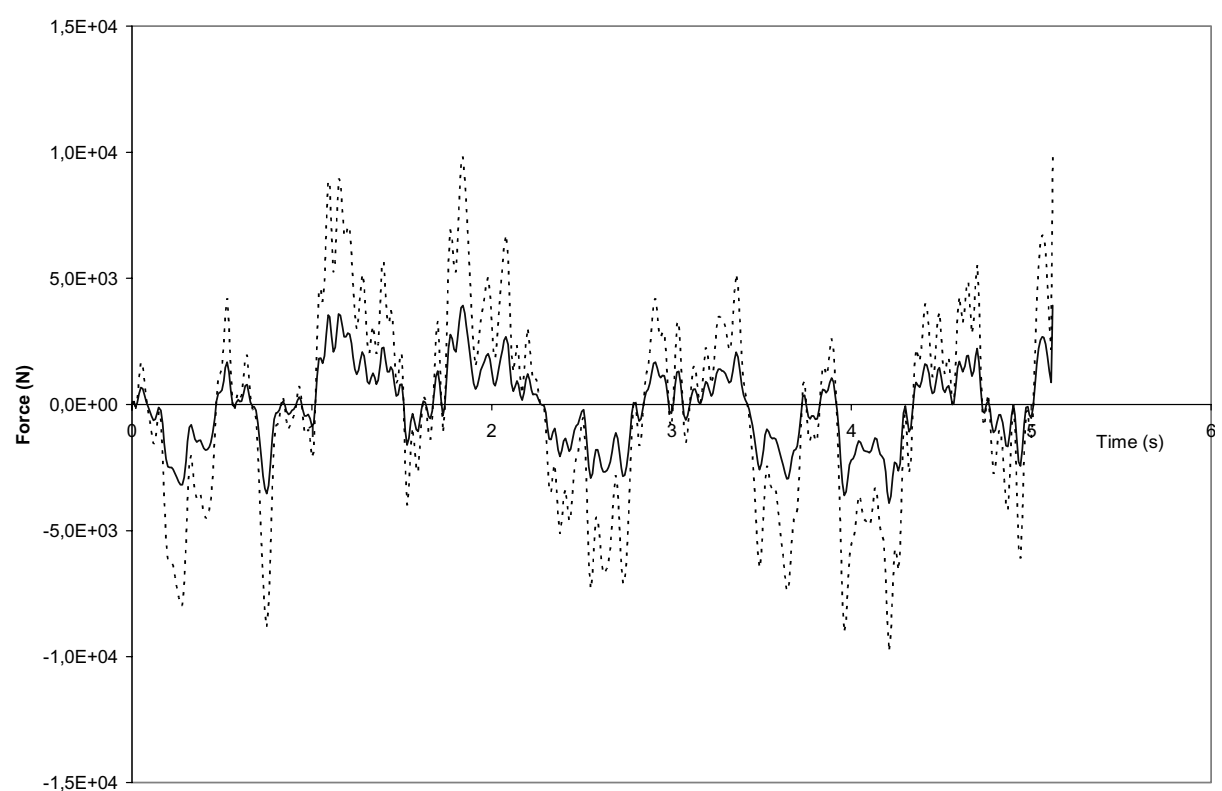

(a) Road excitation F2 in undamped (---) and damped modes (-)

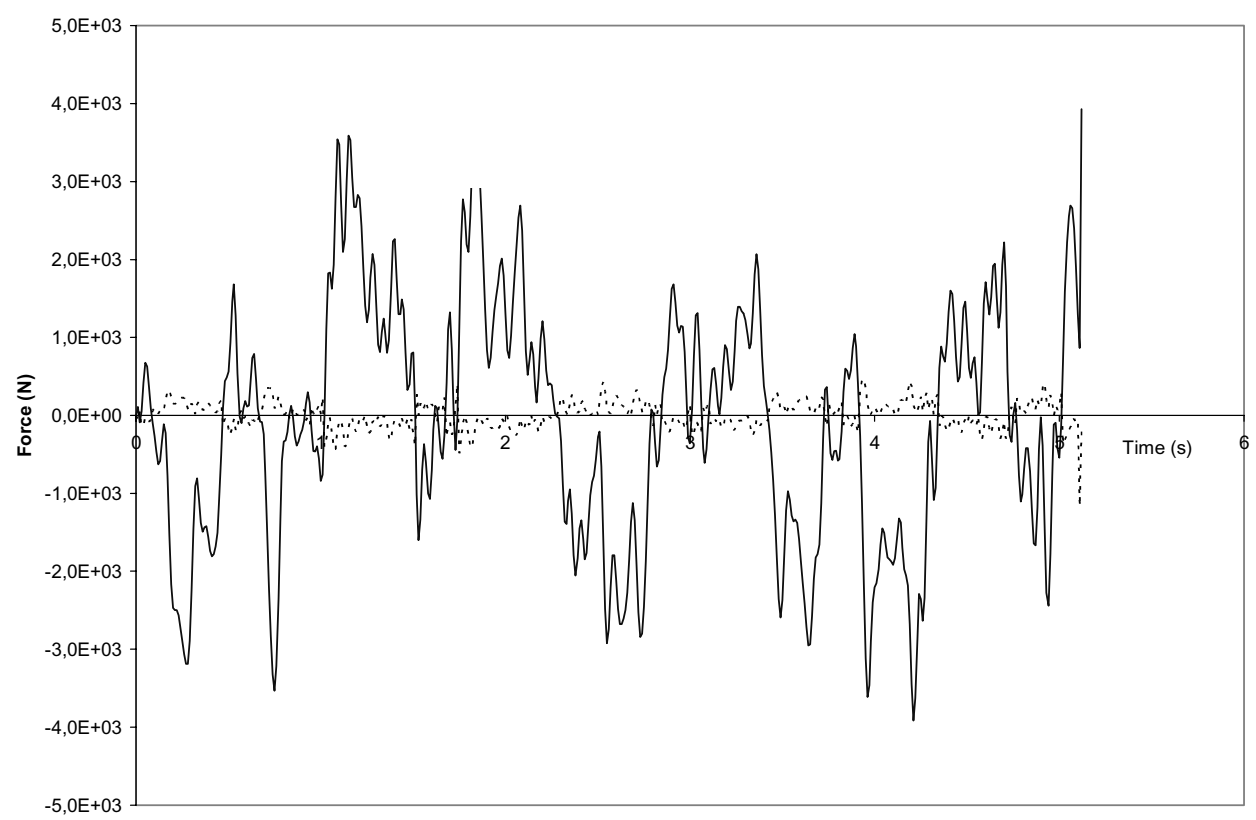

(b) Damped mode: road excitation $F_{2}(-)$ and feedback chain $F_{1}(--)$

Fig. 4. Different force spectrums in the vehicle control suspension system.

numerical diagram of integration depends on the stability of the diagram, the computing time and the precision of the method. We use an implicit integration scheme $[17,18]$. The loading and boundary conditions specify the places of excitation and the degrees of freedom imposed on the system. The mesh takes into account the state of the stress and the point sources, such as the places of loading and the boundary conditions. The uniaxial equivalent is calculated, because both uniaxial and multiaxial models have the same tensor order (order zero) in the fatigue case. This would not be the same for a static case. The critical element is filtered through all the elements of the mesh, giving the maximum sum of the positive variations. The critical element loading signal is corrected. The rainflow cycles are 
extracted using the Markov method. Given the non linearity of the Mansson-Coffin equation, the partial fatigue life is calculated by the Newton method. The number of random cycle repetitions leading to the automobile part's rupture is arrived at the final stage using the Miner law.

To determine the local stress state and the fatigue criterion, Lachowicz [19] used the multiaxial strain energy density criterion without specifying the calculation for the density of plastic deformation energy. Pan et al. [20], Lee et al. [21], and Li et al. [22], compared the experimental results of multiaxial fatigue with models of multiaxial criterion of strain energy density already existing as the criterion of the critical plan which generalizes the Smith Watson Topper (SWT) uniaxial model. The multiaxial case can be studied by calculating the fatigue life of an automobile part in the uniaxial mode by a quantity of strain energy density equivalent to multiaxial case one. The local stress plane state, characterized by: $\sigma_{1}, \sigma_{2}, \varphi_{p}$ and $\lambda=\frac{\sigma_{2}}{\sigma_{1}}$, the two principal stresses, the angle of dephasing between and the local axis $O X$ and the ratio of biaxiality [23], respectively. The stress state in a material can take the following aspects:

- uniaxial case: Both $\lambda=\frac{\sigma_{2}}{\sigma_{1}}=0$ and $\varphi_{p}$ are constant in time. A uniaxial model such as the SWT criterion is used;

- proportional multiaxial case: $\lambda=\frac{\sigma_{2}}{\sigma_{1}}$ varies in time and $\varphi_{p}$ is constant. The effective strain criterion is used;

- nonproportional multiaxial case: $\lambda \stackrel{\sigma_{1}}{\sigma_{1}}$ Both and $\varphi_{p}$ vary in time. The critical plane is used.

In all three cases the uniaxial criterion equivalent of the multiaxial case is used [2]. Since the aluminium alloys present a plastic rather than an elastic behaviour, the nonlinear profile is given by the Ramberg-Osgood equation as:

$$
\varepsilon=\frac{\sigma}{E}+\left(\frac{\sigma}{k^{\prime}}\right)^{1 / n^{\prime}}
$$

where $K^{\prime}=\frac{\sigma_{f}^{\prime}}{\varepsilon_{f}^{\prime n^{\prime}}}$ is the coefficient of the endurance limit, $n^{\prime}=\frac{b}{c}$ the cyclic hardness exponent, the coefficient of the fatigue strain, $c$ the exponent of the fatigue ductility, $b$ the fatigue strain exponent and $\varepsilon_{f}^{\prime}$ the coefficient of the fatigue ductility. The strain energy density relative to the multiaxial case can be expressed as:

$$
U_{s}=U_{\sigma}, \text { or } \int_{0}^{e_{i j}} S_{i j} d e_{i j}=\int_{0}^{\varepsilon_{i j}} \sigma_{i j} d \varepsilon_{i j}
$$

The value of the strain energy density obtained from the analysis in three-dimensional finite element method is made equal to the strain density energy of the uniaxial case that makes up the plastic and elastic deformation energy. The mathematical formulation of the total uniaxial deformation energy per unit volume obtained by an exact integration is represented as:

$$
U_{a}=U_{a e}+U_{a p}=\int_{0}^{\varepsilon_{a}} \sigma_{a} d \varepsilon_{a}
$$

We know that:

$$
\varepsilon_{a}=\frac{\sigma_{a}}{E}+\left(\frac{\sigma_{a}}{K^{\prime}}\right)^{\frac{1}{n^{\prime}}}
$$

thus:

$$
\frac{d \varepsilon_{a}}{d \sigma_{a}}=\frac{1}{E}+\frac{\sigma_{a}^{\left(\frac{1-n^{\prime}}{n^{\prime}}\right)}}{n^{\prime} K^{\prime} \frac{1}{n^{\prime}}}
$$

Equation (11) becomes:

$$
U_{a}=\int \sigma_{a}\left(\frac{1}{E}+\frac{\sigma_{a}^{\left(\frac{1-n^{\prime}}{n^{\prime}}\right)}}{n^{\prime} K^{\prime} \frac{1}{n^{\prime}}}\right) \sigma_{a}
$$

and finally, we obtain:

$$
U_{a}=\frac{\sigma_{a}^{2}}{2 E}+\frac{\sigma_{a}}{n^{\prime}+1}\left(\frac{\sigma_{a}}{K^{\prime}}\right)^{\frac{1}{n^{\prime}}}
$$




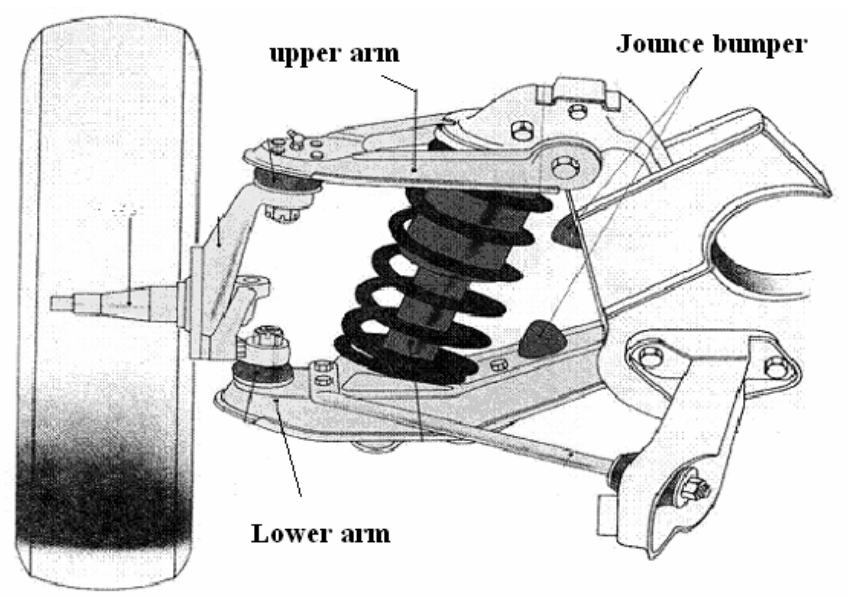

Fig. 5. Upper and lower suspension arms of a vehicle [24].

The solution of Eq. (13) gives the necessary constraint to produce the same energy density as in the uniaxial case. Replacing the stress value in Eq. (10) gives the corresponding deformation. Knowing the deformation, fatigue life can be predicted by using the Manson-Coffin relationship as in Eq. (14):

$$
\frac{\Delta \varepsilon}{2}=\frac{\sigma_{f}^{\prime}}{E}(2 N)^{b}+\varepsilon_{f}^{\prime}(2 N)^{c}
$$

where $\Delta \varepsilon=\varepsilon_{\max }-\varepsilon_{\min }$ is the interval of deformation, $\sigma_{f}^{\prime}$ the coefficient of the fatigue strain, $N$ the number of cycles to failure, $E$ the Young modulus, $C$ the exponent of the fatigue ductility, $b$ the exponent of the fatigue strain and $\varepsilon_{f}^{\prime}$ the coefficient of the fatigue ductility.

\section{Development of optimization algorithm based on strain energy density}

Currently, the lower arm suspension of a vehicle is generally manufactured from steel. Figure 5 illustrates a vehicle suspension containing two suspension arms. The applicability of a weight optimization algorithm to this part already in service is verified. We propose a direct method of weight optimization to obtain a lighter automobile part with a safer fatigue life and a natural frequency away from the PSD frequency range. The optimization is achieved by removing elements of the automobile part that have the minimum energy density sum variation.

We developed a strategy that determined the critical elements and their coordinates, and allowed the isolation of the element with the maximum sum of positive variation of the strain density energy. Contrary to the static case, the rough maximum value of the strain energy density does not necessarily occur in the critical element. Our method allows the application of the Newton-Raphson algorithm to only one element instead of applying it to all the mesh points in the structure. In order to extract the number of cycles to rupture, the nonlinear Mansson-Coffin equation is used. This filter generalizes the case where the excitation is multipoint and shifted in time, giving a tangle of the mesh material element signals. This filter is based on the following algorithm:

$\mathrm{x}_{\mathrm{C}}$ and $\mathrm{z}_{\mathrm{c}}$ are the critical element coordinates having :

$M\left(i_{c}, j_{c}\right)=M_{n c}\left(\operatorname{Max}\left(\sum_{m=0}^{t f} \Delta U(m, n)\right)\right)$

where:

$m=$ time parameter

$k=$ simulation duration

$t f=C_{k}^{2}=\frac{k !}{2 !(k-2) !}$

$n=$ spatial parameter to make $i_{c}$ and $j_{c}$ in the same column $n_{c}$

$\Delta U(m, n)=U(l, n)-U(p, n)$, when $U(l, n)>U(p, n)$ 


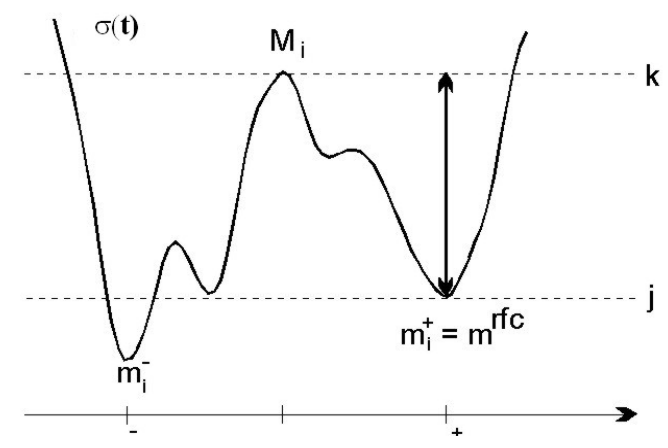

Fig. 6. Mathematical characterization of a rainflow cycle.

Unlike the static case where the absolute value of strain energy causes rupture through fatigue, it is the variation of the strain energy density which causes rupture by fatigue in the dynamic stress case. To apply the Miner law, the rainflow cycle must be extracted. The problem of the direct passage of the PSD of $\sigma(\mathrm{t})$ to the counting of the rainflow cycles is then solved by the rigorous application of a theorem based on the definition of a rainflow cycle [25] and on the Markov chain theory. A rainflow cycle, as illustrated in Fig. 6 can be mathematically characterized in the following way: let us consider $\sigma(\mathrm{t})$ where $t \in[0, T]$ and the stress maximum $M_{i}$ of level $K$ occur at time $t_{i}$. The limits $\left(m_{i}^{-}, M_{i}\right)$ and $\left(M_{i}, m_{i}^{+}\right)$can be defined where:

- $m_{i}^{-}$is the minimum of $\sigma(\mathrm{t})$ and $M i$ is between the last passage to the negative slope of $\sigma(t)$ and the maximum $M i$. This minimum is on the left of $M i$ and occurs at time $t_{i}^{-}$

- $m_{i}^{+}$is the minimum $\sigma(\mathrm{t})$ which is between $M i$ and the first passage to positive slope of $\sigma(\mathrm{t})$ by the level $K$. This minimum is on the right of $M i$ and occurs at time $t_{i}^{+}$.

If there is no passage of $\sigma(\mathrm{t})$ by the level $K$ before or after time $t_{i}$, then $t_{i}^{-}=0$ and $t_{i}^{+}=T$. The rainflow extracted at time $t_{i}$ is then defined either as the extent $\left(m_{i}^{r f c}, M_{i}\right)$, or $\left(M_{i}, m_{i}^{r f c}\right)$. This minimum $m_{i}^{r f c}$ is given by applying the condition:

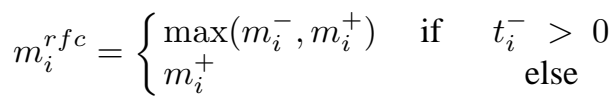

Anomalies which may be present in the strain energy density signal of the critical element can be avoided if the rainflow cycles are counted using the Markov method. Successive equalities of two values of strain energy density at consecutive moments could be a problem. The anomaly is corrected by the WAFO Matlab interface dat $2 \mathrm{tp}$.

When the loadings are composed of cycles of various amplitudes and different average values, it is necessary to measure the total damage produced by these cycles. Fatemi and Yang [25] present a complete review of the laws of damage calculation which were developed from the linear damage rule suggested by Palmgren. The mathematical formulation under which it is currently known was proposed by Miner and it is expressed as Eq. (16):

$$
D=\sum_{i=1}^{n} \frac{n_{i}}{N_{i}}
$$

Random stress history is described as a sequence of blocks of constant amplitude. Each block $i$ is composed of $N_{i}$ cycles of amplitude. Partial fatigue life $N_{i}$ corresponding to this stress amplitude is determined from the Wöhler curve or using the strain energy density approach. Failure is predicted when damage $D$ is equal to 1 . It is thus necessary to find the number of times the random loading is repeated before $D$ is equal to 1(?). It is necessary to find the $B_{f}$ number which one must multiply by $D$ to reach rupture. $B_{f}$ is calculated using Eq. (17):

$$
B_{f}=\frac{1}{\sum_{i} \frac{n_{i}}{N_{i}}} \text { because } B_{f} D=B_{f}\left(\sum_{i} \frac{n_{i}}{N_{i}}\right)=1
$$




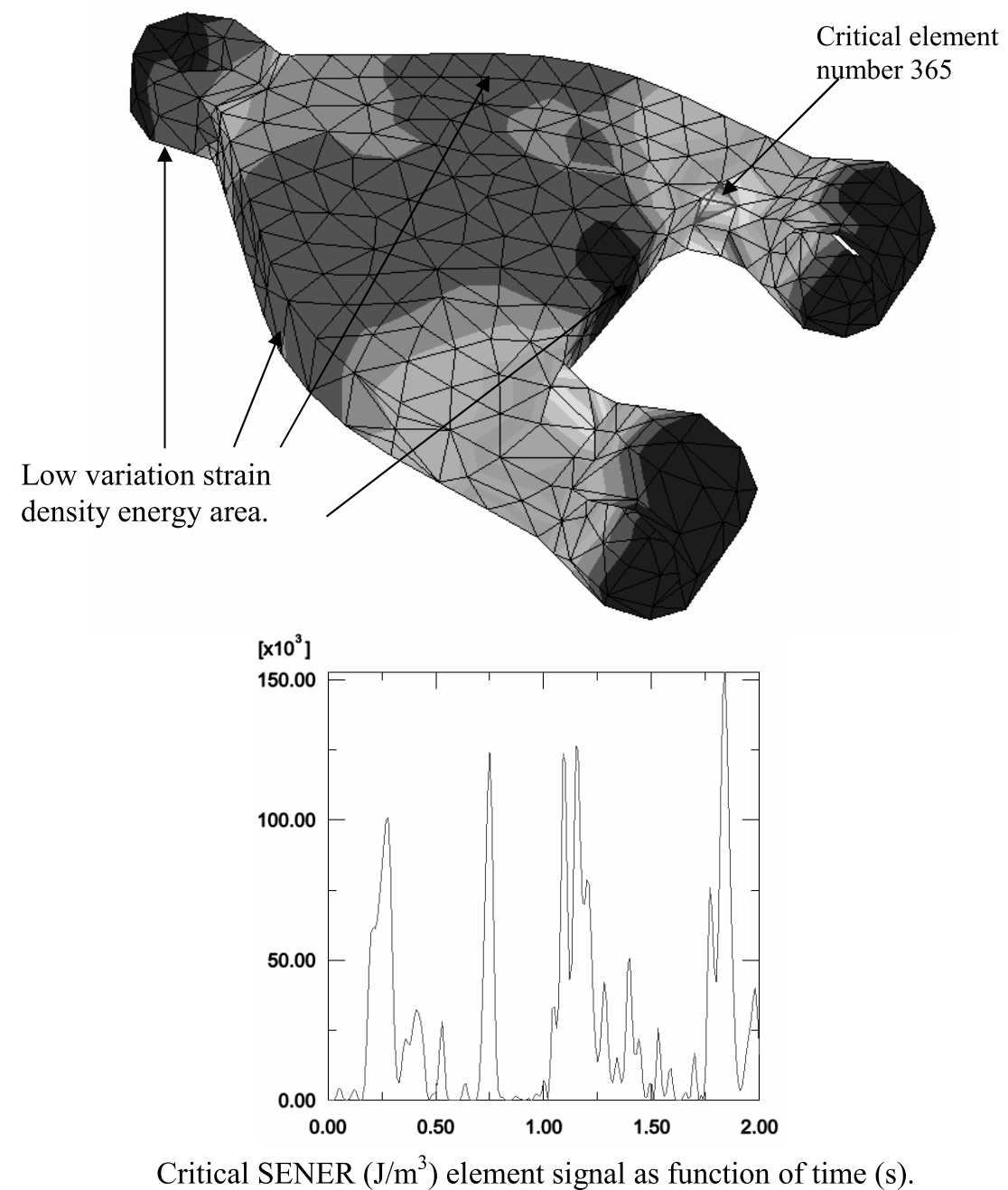

Fig. 7. SENER profile and critical SENER element signal of reference geometry.

In this study the number of cycles to rupture is calculated from the density of uniaxial strain energy (SENER from $S=$ strain, ENER = energy) equivalent of the multiaxial case. SENER reaches a maximum value of $150 \mathrm{~J} / \mathrm{m}^{3}$. The shortest fatigue life number $N R$ of the part is calculated using the Miner law to be $5.2 \times 10^{7}$ cycles caused by continuous excitement. The part weighs about $3.5 \mathrm{~kg}$. Low strain density energy areas are shown in Fig. 7. An initial analysis already enables us to identify areas with low SENER variation. These areas can be removed without affecting the part's performance or reducing significantly its life expectancy or its natural frequencies. Abaqus 6.4 software, calculates natural frequencies based on the theory of elasticity and uses a deformable body such as a non negligible mass spring. Frequency analysis of the most complex structures is laborious and depends on complex system conditions such as: boundary conditions, static equilibrium conditions of the excited system and system mass distribution.

In this paper, the static equilibrium state as an embedded system where the specimen is forced to no motion in the six degrees of freedom and elasticity is due to specimen weight. The first two modes have frequencies of $416 \mathrm{~Hz}$ and $1754 \mathrm{~Hz}$, respectively (Table 3). The reference geometry weight is around $35 \mathrm{~kg}$. The reference geometry of the first two modes is far from the PSD frequency range describing the road profile. Figure 7 shows critical areas as well as areas of low SENER variation. It is known that natural frequencies are proportional to flexural rigidity and inversely proportional to part mass as described above. 
Table 3

Mass and frequencies of the first two modes of the reference geometry

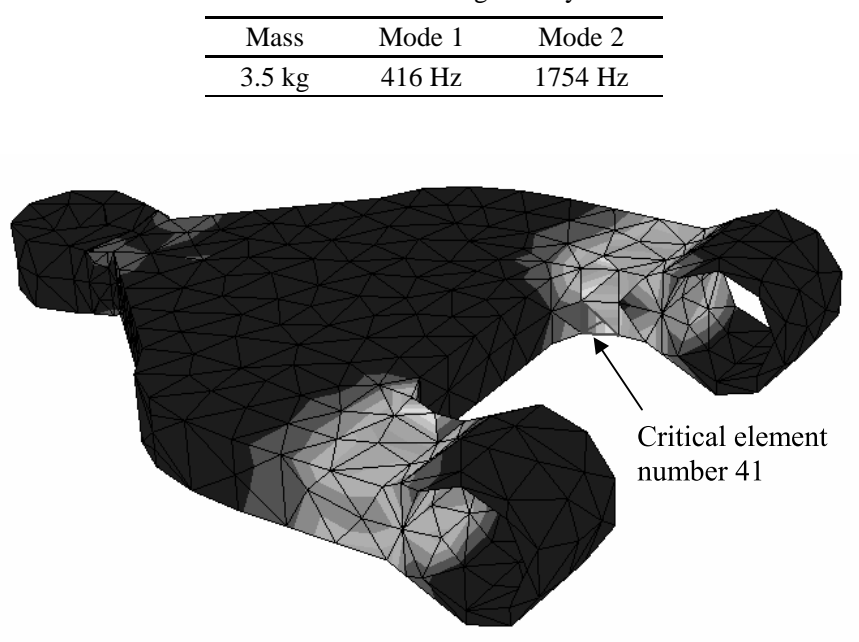

(a) Mass: $3.34 \mathrm{~kg}$. Fatigue life: $10^{8}$. Mode 1: $439 \mathrm{~Hz}$.

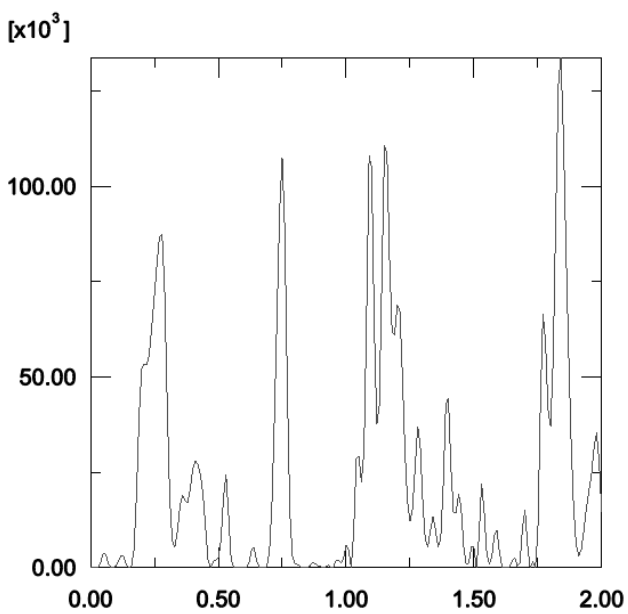

b) Critical SENER $\left(\mathrm{J} / \mathrm{m}^{3}\right)$ element signal as function of time (s).

Fig. 8. First optimization step.

As material in low intensity SENER variation areas is removed. The part's geometry changes and its mass is reduced (Fig. 8). The element removal is symmetrical and must respect the objective function:

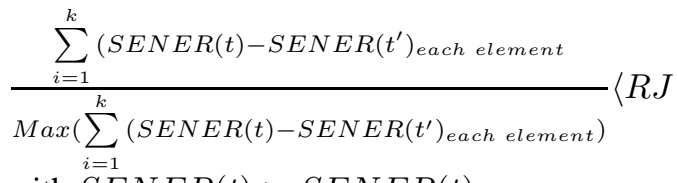

with $S E N E R(t)>S E N E R(t)$

$t$ and $t^{\prime}$ are time parameters

$n=$ number of mesh elements

$k=\frac{n !}{2 !(n-2) !}$ 


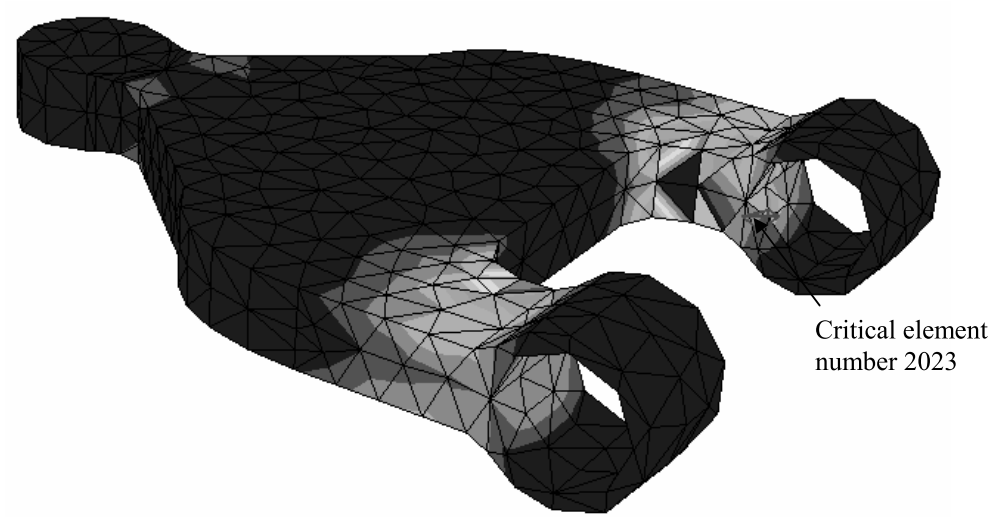

(a) Mass: $3.32 \mathrm{~kg}$. Fatigue life: $2 \times 10^{7}$. Mode 1: $440 \mathrm{~Hz}$.

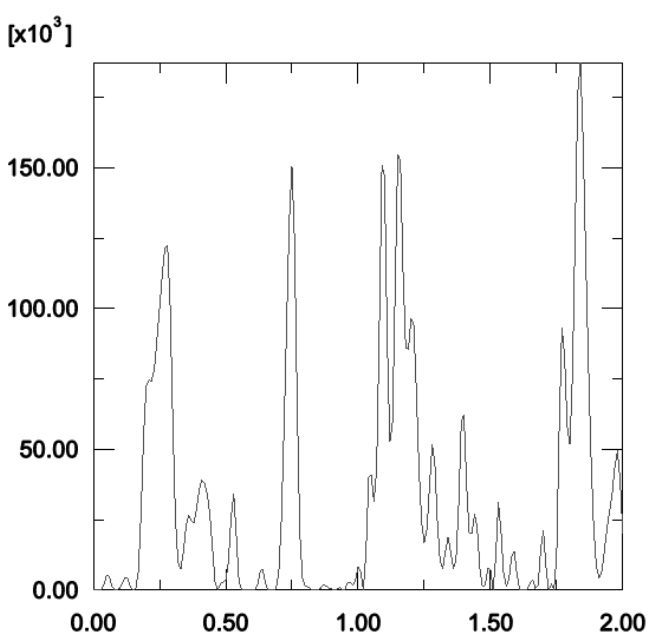

b) Critical SENER $\left(\mathrm{J} / \mathrm{m}^{3}\right)$ element signal as function of time (s).

Fig. 9. Second optimization step.

The rejection ratio $R J$ is directly linked to the notion of fatigue. In order to avoid the extraction of rainflow cycles, $R J$ takes into account the SENER variation, simplifies calculations and adjusts to loading randomness. It also takes into account fatigue life calculations in all mesh elements and optimizes the part's weight in a reasonable time period. SENER fluctuations are due to mesh ratio variation, directly linked to the integration implicit scheme used in this study. The mass decreases by $4.6 \%$ from $3.5 \mathrm{~kg}$ to $3.34 \mathrm{~kg}$. The first natural frequency mode varies from $416 \mathrm{~Hz}$ to $439 \mathrm{~Hz}$ and the second mode from $1754 \mathrm{~Hz}$ to $1883 \mathrm{~Hz}$, moving away from the frequency range of the PSD (Fig. 8). Predicted fatigue life of the part is about $5 \times 10^{7}$ to $10^{8}$. Two other stages of material removal were carried out to reduce weight. Figures 9 and 10 show second and third stage optimization results. The part's mass decreases without affecting either its fatigue life or its first mode significantly. The mass decreases from $3.5 \mathrm{~kg}$ to $3.1 \mathrm{~kg}$, which corresponds to a $11.42 \%$ weight loss. All critical elements are located in the embedded area. Figure 11 shows the geometrical evolution of the part during the optimization process. Numerical results are summarized in Table 4.

The results of the optimization study are comparable to Haiba et al.'s [1] work on weight optimization of a simple mechanical part. However, their fatigue study is based primarily on simple experimental method of the Wöhler curve extrapolation in the calculation of fatigue life. They took into account only one parameter: stress variation. Our approach takes into account two parameters: stress and strain, using the multiaxial criterion of the strain energy density equivalent to the uniaxial case. Haiba et al. [1] defined the fatigue rejection ratio as: $\frac{(\text { Fatigue life })_{\min }}{(\text { Fatigue life })_{\max }}<R R$ where (Fatigue life $)_{\text {min }}$ and (fatigue life $)_{\max }$ are the minimum and maximum fatigue life of 


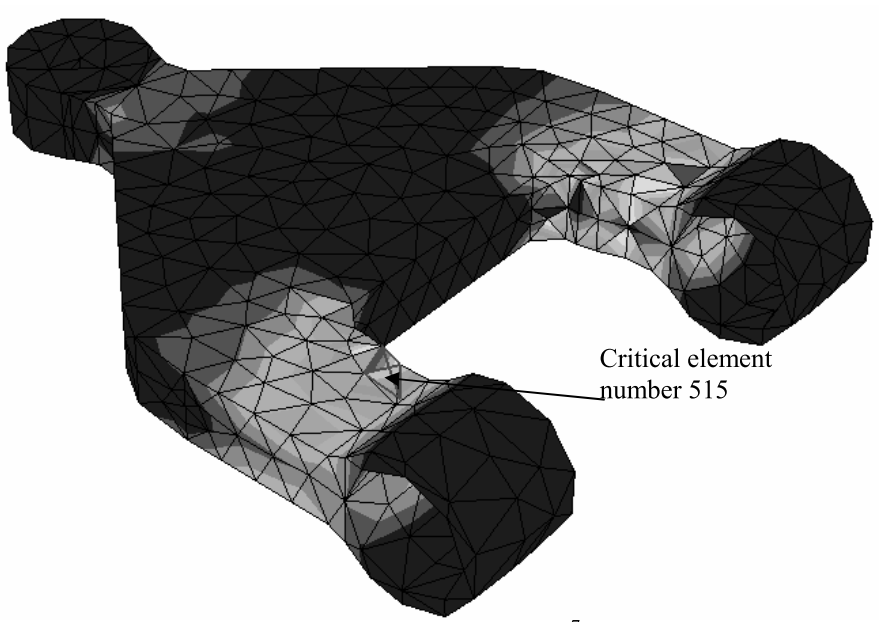

(a) Mass: $3.1 \mathrm{~kg}$. Fatigue life: $5.2 \times 10^{7}$. Mode 1: $438 \mathrm{~Hz}$.

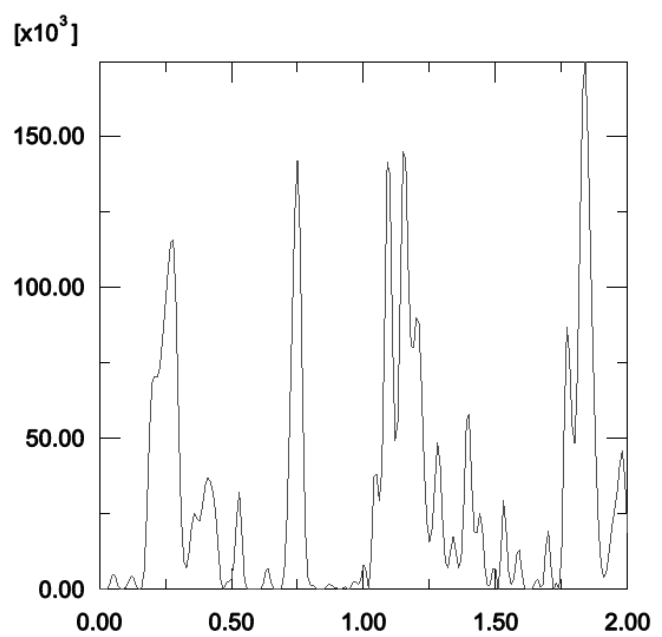

b) Critical $\operatorname{SENER}\left(\mathrm{J} / \mathrm{m}^{3}\right)$ element signal as function of time (s).

Fig. 10. Third optimization step.

the structure, respectively. In their study the critical areas can be strengthened by a detailed optimization study using genetic algorithms and by the use of the rejection ratio developed in the present study. Our proposed optimization approach for making parts lighter is analogous to the method of Haiba et al. [1]. Unlike in Haiba et al.'s [1] work, the rejection ratio developed in the present study is linked directly to the notion of fatigue. It calculates the fatigue life of all mesh elements, and uses a more rational criterion of the strain density energy. The rejection ratio developed here simplifies the calculations and adapts to load random aspect. It avoids rainflow cycle extraction and fatigue life calculation in all elements. It also allows the optimization of a part's weight compared to its fatigue life and its eigen mode in a reasonable time.

\section{Conclusion}

In this study, an analytical and numerical model is worked out to simulate the possible dynamic behaviour of a suspension system as well as the state of the stress and the strain energy density in a lower suspension arm. The multiaxial criterion of the uniaxial case of strain energy density equivalent to the multiaxial one is used to calculate 
Table 4

Numerical results summary

\begin{tabular}{lcccc}
\hline Part & Mass in kg & Fatigue life & Mode 1 & Mode 2 \\
\hline Reference geometry & 3.5 & $5.2 \times 10^{7}$ & $416 \mathrm{~Hz}$ & $1754 \mathrm{~Hz}$ \\
First optimization step & 3.34 & $10^{8}$ & $439 \mathrm{~Hz}$ & $1883 \mathrm{~Hz}$ \\
Second optimization step & 3.32 & $2 \times 10^{7}$ & $440 \mathrm{~Hz}$ & $1889 \mathrm{~Hz}$ \\
Third optimization step & 3.1 & $5.2 \times 10^{7}$ & $438 \mathrm{~Hz}$ & $1861 \mathrm{~Hz}$ \\
\hline
\end{tabular}

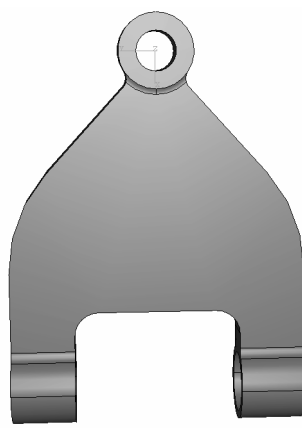

Reference real part : Mass: $3.5 \mathrm{~kg}$ Fatigue life: $5.2 \times 10^{7}$ Mode 1: $416 \mathrm{~Hz}$

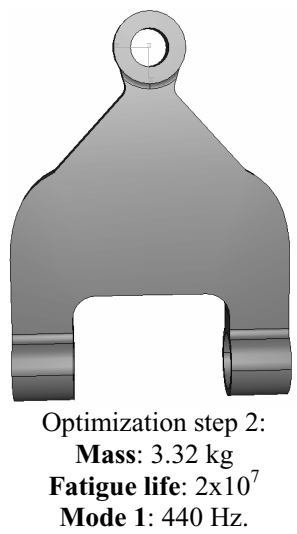

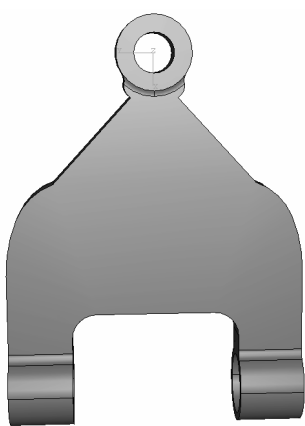

Optimization step 1:

Mass: $3.34 \mathrm{~kg}$

Fatigue life: $10^{8}$

Mode 1: $439 \mathrm{~Hz}$

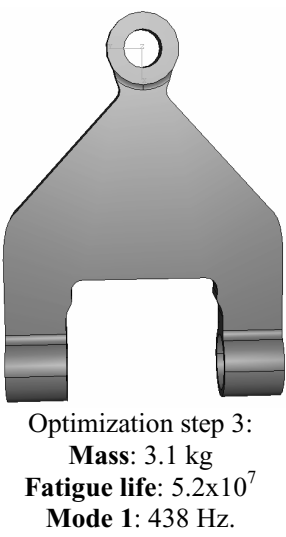

Fig. 11. Optimization process: shape changes in the automobile part.

the part fatigue life. A model of the negative feedback and forward path of the vehicle's lower suspension arm, which is subjected to very great dynamic stress, is developed. Using this model, the maximum force of impact between the road and the tire of a vehicle measured in a 0.5 second fraction is estimated at $10 \mathrm{KN}$. However, the maximum value of the force transmitted by the tire is about $4 \mathrm{KN}$. Numerical calculations show that the maximum negative feedback force is $1200 \mathrm{~N}$ in the damping case and $3750 \mathrm{~N}$ in the rigid case.

To filter the critical element and to extract the fatigue life, a Matlab interface is generated to locate automatically the critical elements without applying the Newton-Raphson algorithm in every element of the mesh. This filter takes into account the multipoint excitation shifted in time. The shifted excitation gives a tangle of the stress signals of the material mesh elements. The elasto-plastic nonlinear case is described by applying the Ramberg-Osgood uniaxial relation binding stress to deformation.

Following the first iteration, all elements with a fatigue life less than $10 \%$ of the critical element are removed; between 5 and $11 \%$ of the weight is lost. Unlike previous methods, the rejection ratio developed in this work is directly linked to the notion of fatigue. This method allows the calculation of the optimum weight for the part in relation to its fatigue life and its eigen mode in a reasonable time period using either a coarse or a refined mesh. 


\section{Acknowledgments}

The work done in this study is sponsored by the Natural Science and Engineering Research Council of Canada (NSERC), CQRDA, FUQAC and CURAL.

\section{References}

[1] M. Haiba, D.C. Barton, P.C. Brooks and M.C. Levesley, Evolutionary structural optimisation of Dynamically loaded components in consideration of fatigue life, Advances in Engineering Software 36 (2005), 49-57.

[2] A. Elmarakbi, H. El-hage and S. Bhattacharjee, Multiaxial fatigue crack initiation by strain energy density using finite element method, CSME Forum, Kingston, May 21-24, 2002.

[3] W.V. Mars, Cracking energy density as a predictor of fatigue life under multiaxial conditions, Rubber Chemistry and Technology, Akron (Mar/Apr 2002), 1-17.

[4] A. Ray, S. Tangirala and S. Phoha, Stochatic modeling of fatigue crack propagation, Applied Mathematical Modeling 22 (1998), 197-204.

[5] A. Ray and R. Patankar, A stochastic model of fatigue crack propagation under variable-amplitude loading, Engineering Fracture Mechanics 62 (1999), 477-493.

[6] A. de-Andrés, J.L. Pérez and M. Ortiz, Elastoplastic finite element analysis of three-dimensional fatigue crack growth in aluminium shafts subjected to axial loading, International Journal of Solids and Structures 36 (1999), 2231-2258.

[7] Thomson, William Tyrell "Theory of vibration with application", (4th ed.), by Prentice Hall, Englewood Cliffs, New Jersey 07632, 1993, 546.

[8] C. Athanasios and A. Chris, Papadopoulos, Identification of multiple cracks in beams under bending, Mechanical System and Signal Processing 20 (2006), 1631-1673.

[9] N.G. Stephen and Y. Zhang, Coupled tension-torsion vibration of repetitive beam-like structures, Journal of Sound and Vibration 293 (2006), 253-265.

[10] H. Salarieh and M. Ghorashi, Free vibration of Timoshenko beam with finite mass rigid tip load and flexural-torsional coupling, International Journal of Mechanical Sciences 48 (2006), 763-779.

[11] E. Hamed and Y. Frostig, Natural frequencies of bonded and unbonded prestressed beams-prestress force effects, Journal of Sound and Vibration 295 (2006), 28-39.

[12] A. Kyprianou, J.E. Mottershead and H. Ouyang, Structural modification Part2: assignment of natural frequencies and antiresonances by added beam, Journal of Sound and Vibration 284 (2005), 267-281.

[13] H. Rahnejat, Multi-Body-Dynamics: vehicles, machines and mechanisms, Society of Automotive Engineers (1998), 355.

[14] M. Bouazara, Étude et analyse de la suspension active et semi-active des véhicules routiers, Ph.D. thesis, Laval University, Quebec, Canada, 1997, 197.

[15] M.G. Owolabi and N.K. Sing, A comparison between analytical models that approximate notch-root elastic-plastic strain-stress components in two-phase, particule-reinforced, metal matrix composites under multiaxial cyclic loading: Theory, International Journal of Fatigue $\mathbf{2 8}$ (2006), 910-917.

[16] M.G. Owolabi and N.K. Sing, A comparison between analytical models that approximate notch-root elastic-plastic strain-stress components in two-phase, particule-reinforced, metal matrix composites under multiaxial cyclic loading: experiments, International Journal of Fatigue 28 (2006), 918-925.

[17] J.S. Sun, K.H. Lee and H.P. Lee, Comparison of implicit and explicit finite element methods for dynamic problems, Journal of Materials Processing Technology 105 (2000), 110-118.

[18] N. Rebelo, J.C. Nagategaal and L.M. Taylor, Comparison of implicit and explicit finite element methods in the simulation forming processes, Numerical Methods in Industrial Forming Processes: Proceedings 4th International (1992), 99-108.

[19] C.T. Lachowicz, Calculation of the elastic-plastic strain energy density under cyclic and random loading, International Journal of Fatigue 23 (2001), 643-652.

[20] W.F. Pan, C.Y. Hung and L. Chen, Fatigue life estimation under multiaxial loadings, International Journal of Fatigue 21 (1999), 3-10.

[21] B.L. Lee, K.S. Kim and K.M. Nam, Fatigue analysis under variable amplitude loading using an energy parameter, International Journal of Fatigue 25 (2003), 621-631.

[22] B. Li, L. Reis and M. De Freitas, Simulation of cyclic stress/strain evolutions for multiaxial fatigue life prediction, International Journal of Fatigue 28 (2006), 451-458.

[23] M. Haiba, D.C. Barton and P.C. Levesley, The development of an optimization algorithm based on fatigue life, International Journal of Fatigue 25 (2003), 299-310.

[24] Associantion canadienne des automobilistes, "Manuel complet de l'automobile" Élection du reader's Digest canada Firme, ISBN $0888501048,1983,480$.

[25] X. Pitoiset, Méthodes spectrales pour une analyse en fatigue des structures métalliques sous chargements aléatoires multiaxiaux, Ph. D. thesis, 2001, 149. 

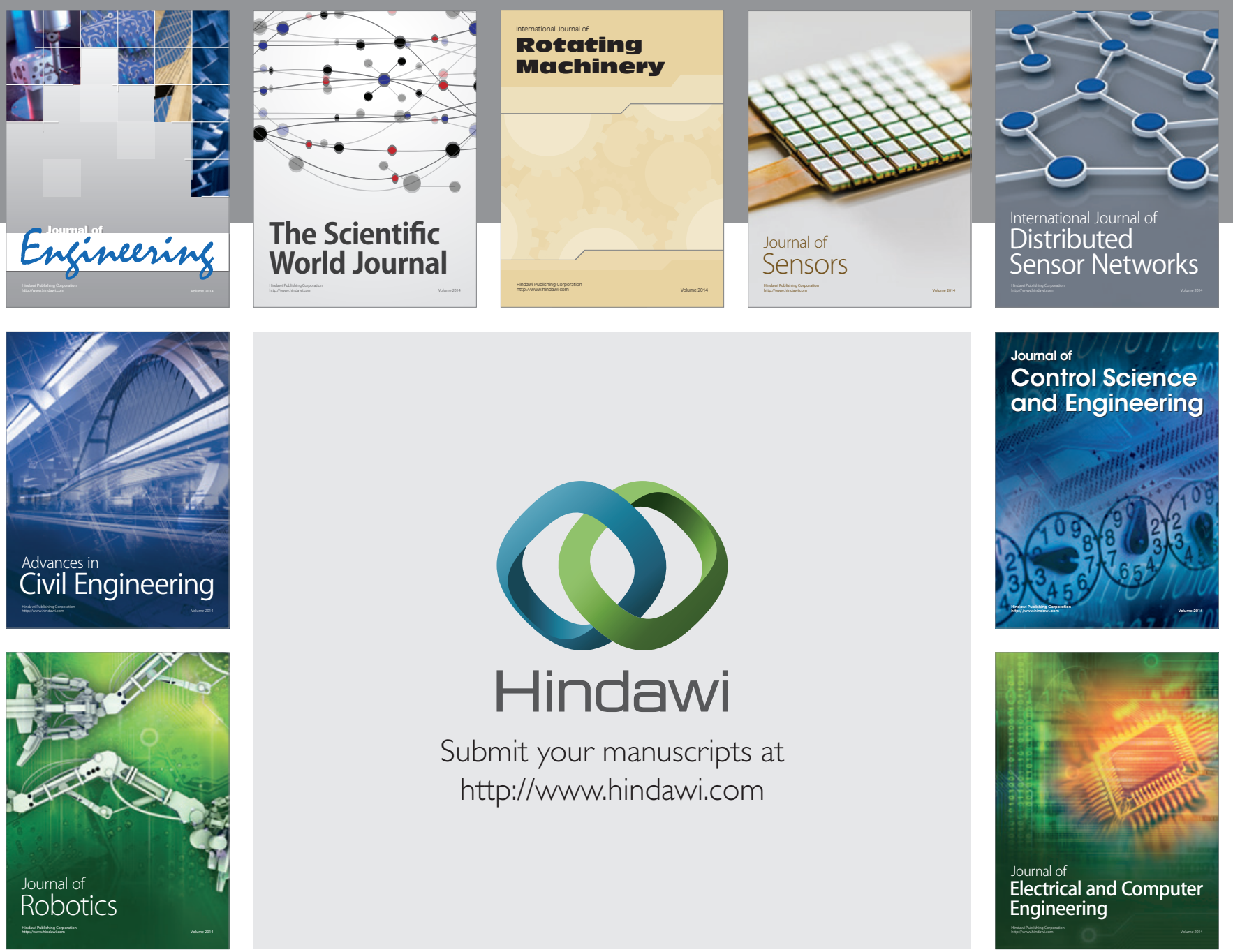

Submit your manuscripts at

http://www.hindawi.com
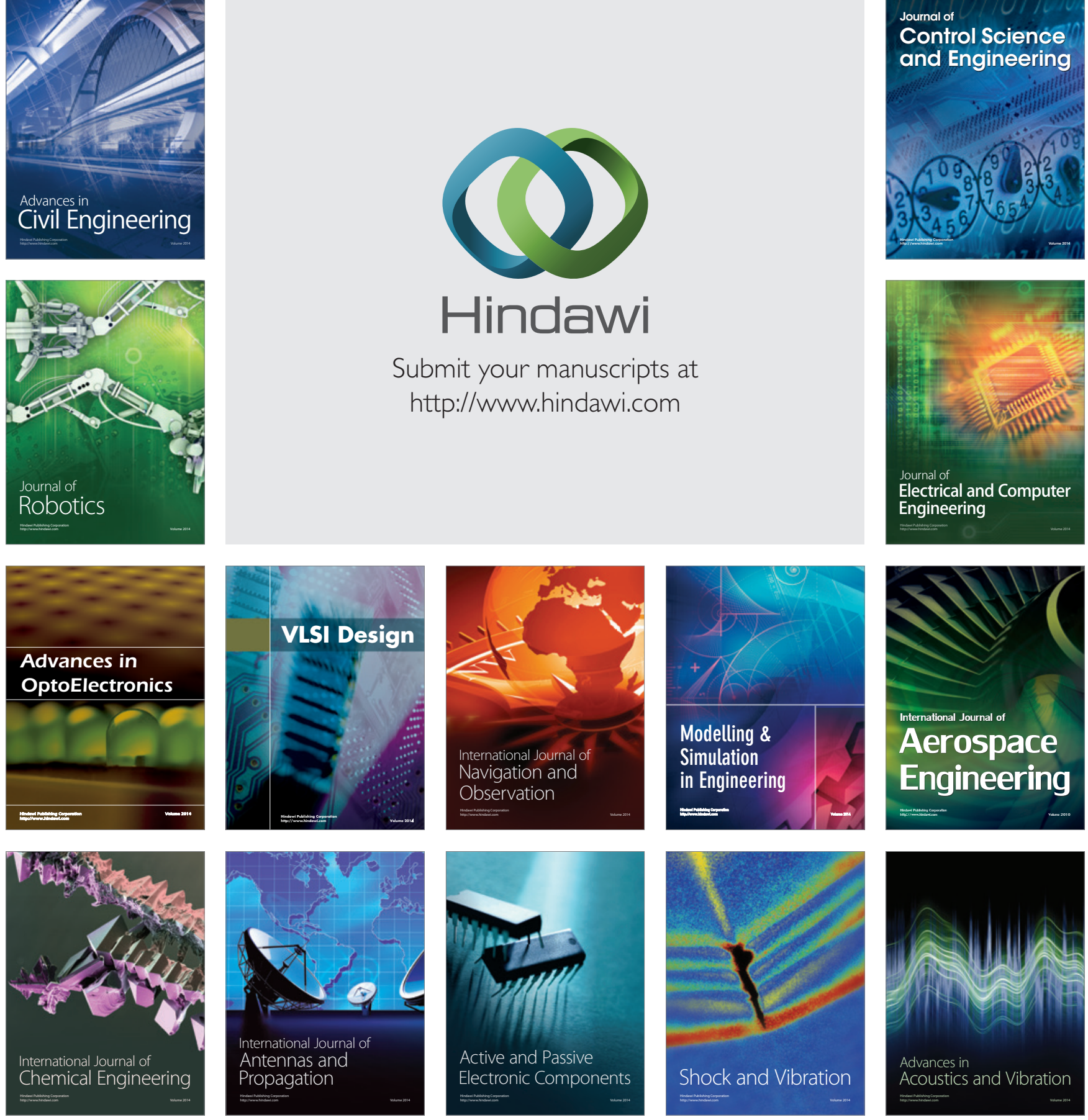